\title{
Improving Microvascular Anastomosis Efficiency by Combining Open-Loop and Airborne Suture
}

\section{Techniques}

\author{
Ahmet Hamdi Sakarya, MD* \\ Division of Reconstructive Microsurgery, Department of Plastic and Reconstructive Surgery, \\ Chang Gung Memorial Hospital, Taoyuan, Taiwan.
}

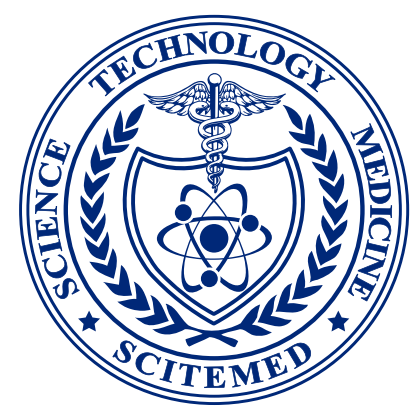

\section{Introduction}

This is a left femoral artery of an adult Lewis Rat weighing 320 grams. The vessel diameter was measured $0.55 \mathrm{~mm}$ (pre-dilatation) and $0.70 \mathrm{~mm}$ (post-dilatation). 16X magnification, 10-0 nylon suture, and 1A vascular double clamp were used. This surgery was performed without assistance. The author acknowledges that any of the techniques illustrated in this video is not new, however, these techniques, which may improve the success rate or ease the anastomosis without assistance, are not commonly used in other centers.

\section{Open-Loop Technique}

In this technique, one can easily use the tips of the forceps to elevate the edge of the vessel $[1,2]$. This elevation not only enables one to see the lumen clearly at all times instead of counting on "feel" but also helps to evert the vessel edges avoiding adventitia and muscle layer inversion to the lumen. The same logic applies to skin suturing while using adson forceps to elevate the skin edges to reduce the angle of a needle entrance for better eversion. Please note that the surgeon is able to use only one tip of the forceps even in very narrow suture intervals during anterior wall repair (thanks to open-loop technique) in order to elevate the right vessel edge for more secure anastomosis with better eversion. Another advantage of this technique is that it speeds up the anastomosis by $25 \%$ [1]. This anastomosis was performed in 8 minutes.

Traction techniques using suture ends generally require assistance to lift the vessel edge and are based on "feel". One can feel the muscle layer without seeing, however, a small piece of adventitia or foreign body that might get in the anastomosis site can be missed. Moreover, if there is a tendency of intimal separation, the traction techniques without a forceps support beneath the intima may cause more separation. The author does not use other techniques unless the position of vessels is exceptionally difficult to perform an anastomosis.

It is notable that the clockwise rotation of the needle holder while pulling out the needle from the vessel followed by the anti-clockwise rotation to hold and reposition the needle back to continue for the next loop.

The author prefers creating smaller loops and leaving the long thread in the first and last loops (as demonstrated in the video) if the vessel size is relatively small, magnification is high, and the space is not wide enough to accommodate the redundant suture that tends to be stuck. However, in free flap surgeries with larger vessels and spaces, the author prefers larger loops and leaving short thread end in the first loop, which makes the anastomosis faster and easier without having to pull suture each time for each loop.

\section{Airborne Suture Technique}

This technique has no use in improving the patency rate; however, it speeds up the anastomosis by preventing the suture from sticking the surrounding tissue [3]. This technique is much easier to apply in less magnification with larger sutures. The author suggests practicing in these conditions first. The long thread is generally preferred to be twice as long in length as the short thread [3]. If the lengths of both threads are similar, more vertical use of the left forceps with more rotation is needed. Right forceps approximate the thread to the relatively stable left forceps. Left forceps do a rotation movement to grab the suture again.

Airborne suture technique is especially useful when there is no one for assistance. The tip of the needle can be used to elevate the edge of the vessel to see the lumen clearly and then place the forceps into the lumen without the need for assistance or irrigation. Moreover, this technique can be used to rotate the vessel for corner sutures without the need for assistance. This technique should be applied in a traumatic manner. One should only use the adventitia layer to lift up the vessel and avoid unnecessary trauma.

The conventional technique of microvascular anastomosis relies on interrupted sutures that require assistance for traction. Because of the traction applied and the limited space and intervals between sutures, the surgeon tends to take an inverting bite from the vessel without seeing the lumen clearly at all times during the anastomosis, which, in my view, is the most critical factor for anastomosis patency. The combination of these techniques minimizes the need for an assistant as well as makes the anastomosis safer and faster.

The main limitation of combining these techniques is the learning curve. Airborne suture technique may not be applicable in every anastomosis environment. It is highly affected by the vessel orientation, limited space, deep anastomosis site, very high magnification, and too much irrigation. With the learning curve, one can overcome these problems. Open-loop technique can be easily applied in any difficult anastomosis environment. The most encountered problem is that the open loops tend to twist and get stuck in the early period of the learning curve. One should cover the dry and irregular surface area around the anastomosis site with wet gauze in order to avoid loops twisting.

\section{PCA Technique}

PCA technique (Posterior wall first-Continuous interrupted-Airborne) [4] is another fast and reliable method. The author prefers PCA technique for end-to-side anastomosis and in situations where it is very difficult to turn over the double vascular clamp for the posterior wall, especially in the setting of short vascular stump or limited space; for example, hepatic artery anastomosis, temporal superficial vessel anastomosis, and rib-sparing technique for internal mammary vessel anastomosis. However, the disadvantage of this technique is that the number of loops created in the anterior wall is sometimes too many, which complicates the repair. Moreover, the author prefers to have a better control during the posterior wall repair with adjusted bites in thickness in the setting of size or thickness discrepancy, and hence the direct repair of posterior wall is usually performed after anterior wall repair.

\section{Alternative Techniques}

There are other techniques described in the literature for eversion, such as eversion with four sutures by Turan et al [5]; however, the author has no experience in this suture technique. In the author's experience, taking smaller bites from the thinner vessel and larger bites from the thicker 


\section{HOW I DO IT}

vessel with an acute angle needle entrance avoids intimal flap formation and establishes enough eversion.

Non-suture microvascular anastomosis using anastomotic coupling device is also a fast and reliable method. The main drawback of this technique is that the device is not readily available in some centers. Coupling device is not available in our center now. The cost of the device is another issue. Furthermore, a very recent study shows that small-diameter anastomotic coupling devices for smaller vessels have higher rates of venous thrombosis compared to large-diameter anastomotic coupling devices for large vessels and hand-sewn anastomosis [6].

\section{Video}

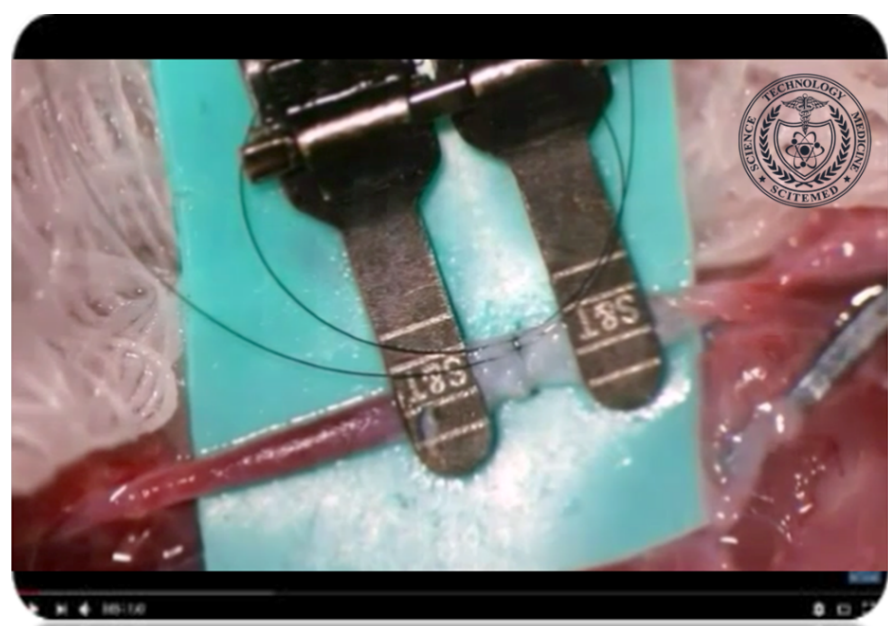

\section{Article Information}

*Correspondence: Ahmet Hamdi Sakarya, MD

Division of Reconstructive Microsurgery, Department of Plastic and Reconstructive Surgery, Chang Gung Memorial Hospital, Taoyuan, Taiwan. Email: dr.ahmetsakarya@gmail.com

Received: Oct. 02, 2017; Accepted: Dec. 05, 2017; Published: Jul. 14, 2018

DOI: 10.24983/scitemed.imj.2018.00069

Copyright $\odot 2018$ The Author(s). This is an open-access article distributed under the terms of the Creative Commons Attribution 4.0 International License (CC-BY).

Funding: None

\section{Conflict of Interest: None}

\section{Keywords}

Airborne suture; anastomosis; open-loop suture.

\section{References}

1. Lee S, Hweidi SA, Skivolocki WO. An open-loop technique to facilitate microtubal anastomosis in the rat. J Reconstr Microsurg1984;1(1):45-48.

2. Yun-Huan Hsieh C-HL, Soo-Ha Kwon, Tommy Nai-Jen Chang. Chang's Technique of Sequential End-to-Side Microvascular Anastomosis Technique of sequential end-to-side anastomosis. International Microsurgery Journal 2017;1(1):4.

3. Chen HC, Mardini S, Tsai FC. "Airborne" suture tying technique for the microvascular anastomosis. Plast Reconstr Surg 2004;113(4):12251228.

4. Cigna E, Curinga G, Bistoni G, Spalvieri C, Tortorelli G, Scuderi N. Microsurgical anastomosis with the 'PCA' technique. J Plast Reconstr Aesthet Surg 2008;61(7):762-766.

5. Turan T, Ozcelik D, Kuran I, et al. Eversion with four sutures: an easy, fast, and reliable technique for microvascular anastomosis. Plast Reconstr Surg 2001;107(2):463-470.

6. Hanson SE, Mitchell MB, Palivela N, et al. Smaller diameter anastomotic coupling devices have higher rates of venous thrombosis in microvascular free tissue transfer. Plast Reconstr Surg 2017:140(6):1293-1300. 